\title{
Potential Assessment Multi-Repeating Abiotic/Biotic Motivation Coincide Biofertilizers to Optimize Black Cumin (Nigella sativa L.) Seed Yield Production and Quality
}

\author{
Tarek Elsayed S.A, El Sayed S.A \\ Radioisotopes Department, Nuclear, Research Center, Egyptian Atomic Energy Authority, Cairo, Egypt \\ Email: dr.tarekelsayed64@gmail.com
}

How to cite this paper: S.A, T.E. and S.A, E.S. (2021) Potential Assessment Multi-Repeating Abiotic/Biotic Motivation Coincide Biofertilizers to Optimize Black Cumin (Nigella sativa L.) Seed Yield Production and Quality. Agricultural Sciences, 12, 69-83. https://doi.org/10.4236/as.2021.122006

Received: December 22, 2020

Accepted: February 6, 2021

Published: February 9, 2021

Copyright $\odot 2021$ by author(s) and Scientific Research Publishing Inc. This work is licensed under the Creative Commons Attribution International License (CC BY 4.0).

http://creativecommons.org/licenses/by/4.0/

(c) (i) Open Access

\begin{abstract}
Black cumin (Nigella sativa L.) the highly aggregate valuable medicinal plant was field cultivated for two subsequent seasons $(2018,2019)$ designed as factorial split plot based on randomized complete block with 3 replications. The main factors 4 elicitors: salicylic acid, (SA) Nano-selenium (NPs), yeast (YS) chitosan $(\mathrm{CH})$ and $(\mathrm{E} 0)$, control. Whereas, the sub-main plot 4 biofertilizers, dray Moringa leaves extract, (MLE), neem dray leaves extract (NME), humic acid (HA) and traditional (NPK) chemical fertilizer as control. Allied statistical analysis of variance revealed that biotic and abiotic elicitors coincide biofertilizer and NPK chemical fertilizer actuated significant positive impacts, dray seed, seed fixed oil, seed essential oil yield production. Also, significantly amelioration bioactive major fatty acids content of seed fixed oil (linolenic $>$ carvone) dihydrolenoleic > oleic) as well as major terpens content of seed essential oil (P-cymene $>$ thnymoquione $>$ Penine). Consequently, multi-repeating elicitation cod be considered reliable strategy achieve sustainable development for $N$-sativa under, biotic elicitor coincide biofertilizers that excel abiotic elicitors coincide biofertilizer which excel biotic or abiotic elicitors coincide NPK traditional chemical fertilizer.
\end{abstract}

\section{Keywords}

Blacke Cumin, Medicinal Plant, Elicitation, Salicylic Acid, Chitosan, Nano-Selenium, Yeast, Biofertilizers, Essential Oil

\section{Introduction}

Nigella (Nigella sativum L), is an annual medicinal plant belongs to family 
Raunculaceae, growing in countries, bordering the Mediterranean region (Including Egypt) western Asia countries, including India, Pakistan, Saudi Arabia and Eastern Europe [1] [2]. In traditional remedy, N. sativa (NS) seeds commonly used as a spice and carminative [1]. In addition, several properties such as liver tonics, diuretic, digestive, anti-diarrheal, appetite stimulant, analgesics. NS seed capsule is effective on decreasing the severity of physical symptom of premenstrual syndrome [3]. Also used as anti-cancer drug and protective agent against gamma radiation induced adverse effects in cell line [4].

N. sativa Seed contains more than $30 \%$ fixed oil and $0.45 \%$ essential oil [5]. Thymoquinone (TQ) is an abundant component of black seed essential oil extract [6]. As antidote or a protective agent against natural or chemical toxicities [7]. There are many reports on the biological activities including, antioxidant [8], analgestic, anti-inflammatory [9] [10], antitussive [11], anti-hypertensive [12], anti-diabetic [13], anti-bacterial [14] and anticancer [15] [16]. In addition, the protective effects of $N$. sativa and its main constituents in different tissues including brain [17] [18] [19], heart [20], liver [21], Kideny [8], lung [22] [23], have been established against some toxic agents [7]. This is also revealed that most of therapeutic properties of $N$. sativa are due to the presence of TQ which is major bioactive components of essential oil [24], along with high value component such as linoleic acid, dithymoquinone [4] [25] [26]. It is now well documented that most of therapeutic benefits of NS are mainly attributed to TQ, which is almost one of the key constituents of its volatile oil [4] [16].

Seeds and seed oil of NS as to explore their potential applications for the development of innovative functional food, nutriceutical and pharmaceutical as well as to help scientific basis of the widespread traditional uses of this multipurpose spice [26]. NS fatty oil includes one important fatty acid of linoleic acid which high industrial importance and recognized an essential biochemical component for human diet [27]. In particular, polyunsaturated fatty acids are very important for maintaining biological function in mammalians, hence regarded essential fatty acids [28]. Aside, it clearly recognized that humans and many animals can abstained monounsaturated fatty acids from sugar (existing in the booly) while due to lake of desaturase enzyme, human body is unable to convert oleic acid into linoleic omega 6 and lenolenic (omega 3) [29]. Therefore, humans forced to append these polyunsaturated fatty acids [30]. Polyunsaturated in human are converted again inavaliable molecules such as antioxidants which protect to body [31] [32]. Unsaturated fatty acids are naturally important wherease Oleic acid is the most popular mona-unsaturated fatty acids capable of decreasing cholesterol, linoleic (omega 6) and lenaleinic (omega 3) are polyunsaturated fatty acids which are effective in reducing the risk of blood pressure and vascular diseases [33]. NS seed and oil, potential sourcer of high-value components for development of functional foods and untriceuticals/pharaceuticals [26].

Elicitation; improve quale-quantitative bioactive secondary metabolites (BMS) 
which improve the health pronating qualities [34]-[40]. It also modified growth and development, can have benifical effect on morphological, physiological, biochemical characteristics that increase biomass yield production and quality [41] [42] [43]. Elicitation had distinct role in regulation of plant and pathogen attack, that cause hug loss in yield production under agrochemical. Traditional agriculture [44] which often implicated to induce systemic resistance ISR) by regulating the expression genes involved for production and accumulation SMs phytoalexins $\left(\mathrm{PH}_{\mathrm{S}}\right)$ specific toxins characterized brood spectrum bio-anti-pesticide and microbicides making them improving against microbial diseases and pests infestation [42] [45] [46] [47]. Exceedingly elicitation plays a major role in adaptation of plants to the changing environmental, overcoming biotic/abiotic stresses [48] [49] [50].

Allied on the best of our knowledge, no findings have been reported on multi-repeating elicitation technology for improvement sustainable agriculture medicinal and aromatic plants. Therefore, the present study aimed to evaluate multi-repeating elicitation aside biofertilizers in respect to ameliorate $N$. sati$v a-$ yield production and quality.

\section{Material and Methods}

\subsection{Elicitor Application}

Foliar spray with two abiotic elicitor; (SA) salicylic acid (E1) $50 \mathrm{mg} / \mathrm{L}$, (NPSe) nano-Selenium oxide (E2) $50 \mathrm{mg} / \mathrm{L}$ and 2 biotic elicitors; (YS) yeast, Saccharomyces servisiae, (E3) $150 \mathrm{PPm},(\mathrm{CH})$ chitosan (E4) 25\% with $500 \mathrm{ML}$ acid; with tween $80,0.1 \% \mathrm{w} / \mathrm{v}$. Meanwhile Zero elicitor control (EO) plants were sprayed only with Tween 80 solution.

\subsection{Fertigation Management}

Four fertilizers were applied; NPK 20:20:20 chemical fertilizer, $20 \mathrm{~g} / \mathrm{m}^{2}$ as traditional (F1) control and three biofertilizers, $20 \mathrm{~g} / \mathrm{m}^{2}$ dry moringa (F2) (Moringa olifera L.) dray leaves extract (MOLE), $20 \mathrm{~g} / \mathrm{m}^{2}$ neem (F3) (Azodiracha indica A. Juss) dray leaves extract (NMLE), $20 \mathrm{~g} / \mathrm{m}^{2}$ Humic acid (HA) as (F4).

\subsection{Execute Field Experiment}

Two subsequent field experiment trial, 2018 and 2019, on orchard farm at Agriculture Research center were designed as factorial split plot besed on randomized complete block with three replications. Four elicitors as main plot and 3 biofertilizer (F2 - F4) and NPK (F1) as sub-main plot. N. sativa seeds were sowing 10 October at both two seasons 2018 and 2019 in plots $1.5 \times 2 \mathrm{~m}$. size in rows 40, 20 intra and enter space to form $12.5 \mathrm{plant} / \mathrm{m}^{2}$. Resultant plants aged $60,120,180$ were foliarly sprayed with elicitors (EO, E1 - E4). Irrigation and fertigation managements through drip surface irrigation system. Harvesting during full flowering stage in two subsequent seasons at 10 June 2018 and 2019. 


\subsection{Biometric Growth Traits}

Five randomly selected plants were recorded for plant height, $\mathrm{cm}(\mathrm{PH}, \mathrm{cm})$, number of primary branches (NPBP) number of secondary branches (NSBP), number of capsule (NCP) number of seeds per capsule (NSC), seed yield, $g$ (SYP, g). Means of these traits were subjected to ANOVA statistical analyses.

\subsection{Quali-Quantitative Yield Traits}

\subsubsection{Seed Yield}

Seed yield per plots were recorded that were converted to seed yield, $\mathrm{g} / \mathrm{m}^{2}$.

\subsubsection{Fixed Seed Oil}

Fifty gram of powdered seed sample/plot subjected to soxhlet apparatus with 250 $\mathrm{ml}$. of petroleum ether for $4 \mathrm{~h}$. [51]. The extract was concentrated under reduced temperature and pressure and fatty oil (fixed oil) percentage was computed using the following formula:

$$
\begin{aligned}
& \text { Seed fixid oil } \%(\mathrm{SFO} \%) \\
& =\left[\frac{\text { Weight of oil }}{\text { Weight of sample }} \times 100-\% \text { seed moisture }\right]
\end{aligned}
$$

Fixed seed oil yield, $\mathrm{g} / \mathrm{m}^{2}$ (SFOY, $\mathrm{g} / \mathrm{m}^{2}$ ) was estimated by multiplying (SFO\%) with SY, $\mathrm{g} / \mathrm{m}^{2}$ for each plot. Concerning SFO quality, fatty acid composition were determined as \% by GC analysis using a thermoquest gas chromatography through a flame ionized detector.

\subsubsection{Seed Essential Oil}

Fifty gram seed/plot were subjected hydro-distillation for $3 \mathrm{~h}$. to optain EO content. EO was dried using anhydrous sodium sulfate and kept in amber glass seed with Teflon septra at $4^{\circ} \mathrm{C}$ intel analysis. Essential oil yield content (EOY, g/kg) and $\mathrm{EO}$ yield $\mathrm{kg} / \mathrm{m}^{2}$ were calculated by the following equations.

EOY, g. $/ \mathrm{kg}(\%)=(($ Extracted EO, $\mathrm{g} / 50 \mathrm{~g}$. ground sample $) \times 100-$ moisture seed content $)$

$$
\text { EOY } \mathrm{g} / \mathrm{m}^{2}=\mathrm{SYg} / \mathrm{m}^{2} \times \mathrm{SEO} \%
$$

EO composition was analyzed by GC/MS using on Agilent Technologies 7890 gas chromatograph coupled to quadruple El-mass analysis and Agilent 9575C mass selective detector.

\section{Statistical Analysis}

The data sets were firstly tested for normality by the Anderson and Darling normality test using a statistical analysis system (SAS) (SAS 2003). The pooled mean values of 2 years for all the traits were subjected to statistical analysis of variance was done for all traits. A least significant difference (LSD, 1\%) test was used for mean comparison of treatment. 


\section{Results and Discussion}

\subsection{Growth Traits}

Statistical analysis of variance revealed that malti-repeating elicitation with abiotic elicitors, salicylic acid, SA. (E1), nano-particale selinum, NPSe. (E2) and biotic elicitors; yeast, SY. (E3), chitosan, $\mathrm{CH}$ (E3) coincide. biofertilizers; moringa leaves extract, MOE (F2), neem leaves extract, NME (F3), humic acid, HA (F4) actuated significant promotion on growth traits actuated over that of NPKEo control (Table 1). At such trait, biotic elicitor; $[\mathrm{CH}(\mathrm{F} 4)>\mathrm{YS}(\mathrm{F} 3)]>$ abiotic elicitor, [NPSe (E2) > SA (E1) inseparate with biofertilizers, [MOE (F2) > NME (F3) > HA (F2)] > NPK chemical fertilizer (F1). Extensive investigations were in line concerning biotic/abiotic elicitation premating growth trits under chemical fertilizer system [37] [39] [52] [53]. Whereas, studies have been not available concurring biofertilizers, system with biotic/abiotic elicitation specially for $N$. sativa. However, solitary biofertilizer acted promotion growth traits has been reported [54] [55] [56].

Table 1. Mean growth traits values for 2 years (2018) and (2019) for $N$-sativa in response to 5 elicitors (E1-4) under 4-fertilizers (F1-4).

\begin{tabular}{|c|c|c|c|c|c|c|}
\hline Application (F/E) & $\begin{array}{c}\text { Plant height, } \\
\mathrm{cm} \\
(\mathrm{PH}, \mathrm{cm})\end{array}$ & $\begin{array}{c}\text { No. primary } \\
\text { branch/plant } \\
(\mathrm{NPB} / \mathrm{P})\end{array}$ & $\begin{array}{c}\text { No secondary } \\
\text { branch/plant } \\
(\mathrm{NSB} / \mathrm{P})\end{array}$ & $\begin{array}{l}\text { NO. capsules per } \\
\text { plant }(\mathrm{NC} / \mathrm{P})\end{array}$ & $\begin{array}{l}\text { NO. seed per } \\
\text { capsules } \\
(\mathrm{NS} / \mathrm{C})\end{array}$ & $\begin{array}{c}\text { Dray seed yield, } \\
\text { g/plant } \\
\text { (DSY, g./P) }\end{array}$ \\
\hline \multicolumn{7}{|l|}{ NPK control } \\
\hline F1E0 & $24.31(100)$ & $9.40(100)$ & $19.51(100)$ & $75.15(100)$ & $70.57(100)$ & $25.149(100)$ \\
\hline F1E1 & $26.74(110)$ & $12.50(133)$ & $22.14(114)$ & $79.06(105)$ & $74.10(105)$ & $28.921(115)$ \\
\hline F1E2 & $27.47(113)$ & $13.63(145)$ & $23.75(122)$ & $80.79(108)$ & $76.22(108)$ & $29.676(118)$ \\
\hline F1E3 & $28.44(117)$ & $15.79(168)$ & $25.37(130)$ & $81.99(109)$ & $76.92(109)$ & $30.179(120)$ \\
\hline F1E4 & $29.17(120)$ & $16.45(175)$ & $26.25(135)$ & $84.32(112)$ & $79.04(112)$ & $30.682(122)$ \\
\hline \multicolumn{7}{|l|}{ MOLE } \\
\hline F2E1 & $33.41(138)$ & $18.23(194)$ & $28.33(145)$ & $88.68(118)$ & $83.27(118)$ & $31.436(125)$ \\
\hline F2E2 & $43.16(141)$ & $19.85(211)$ & $29.71(152)$ & $89.43(119)$ & $83.98(119)$ & $31.688(126)$ \\
\hline F2E3 & $35.37(146)$ & $21.35(227)$ & $31.38(161)$ & $90.93(121)$ & $85.39(121)$ & $32.442(129)$ \\
\hline F2E4 & $36.24(149)$ & $23.11(246)$ & $33.15(170)$ & $93.93(125)$ & $88.21(125)$ & $33.699(134)$ \\
\hline \multicolumn{7}{|l|}{ NMLE } \\
\hline F3E1 & $32.88(135)$ & $17.41(185)$ & $27.55(141)$ & $86.42(115)$ & $81.16(115)$ & $31.059(123)$ \\
\hline F3E2 & $33.52(138)$ & $18.95(202)$ & $28.85(149)$ & $87.18(116)$ & $81.86(116)$ & $31.185(124)$ \\
\hline F3E3 & $34.74(143)$ & $20.21(215)$ & $30.67(157)$ & $90.18(120)$ & $48.68(120)$ & $31.939(127)$ \\
\hline F3E4 & $35.66(147)$ & $22.15(236)$ & $32.22(165)$ & $90.93(121)$ & $85.39(121)$ & $32.945(131)$ \\
\hline \multicolumn{7}{|l|}{ HA } \\
\hline F4E1 & $31.74(131)$ & $17.02(181)$ & 27.07 (139) & $85.67(114)$ & $80.45(114)$ & $30.808(122)$ \\
\hline $\mathrm{F} 4 \mathrm{E} 2$ & $32.44(134)$ & $18.33(195)$ & $28.15(144)$ & $86.42(115)$ & $81.16(115)$ & $30.933(123)$ \\
\hline F4E3 & $33.62(138)$ & $19.74(210)$ & $29.85(153)$ & $88.68(118)$ & $83.27(118)$ & 31.185 (124) \\
\hline F4E4 & $34.51(142)$ & $20.78(221)$ & $30.72(158)$ & $89.43(119)$ & $83.93(119)$ & $32.191(128)$ \\
\hline LSD $1 \%$ & 0.12 & 0.06 & 0.15 & 0.56 & 0.42 & 0.096 \\
\hline
\end{tabular}

E0-4; control, salicylic acid (SA), nano-selenium oxide (SeN), yeast (Y), chitosan (CHT), E0 control respectively. F1-4; NPK, Moring a dry leaf extract (MOLE), Neem dry leaf extract (NMLE), humic acid (HA), respectively. Values between parenthesis (were percent of control). 


\subsection{Quantitative Yield Traits}

F1-4 coincide E1-4 performed significant augmentation for DSY, g/m² SFY, g/m $\mathrm{m}^{2}$ and SE0Y, $\mathrm{g} / \mathrm{m}^{2}$ as\% over control (F1E0) as shown (Table 2) and represented Figures 1-3. Aside, it follows that $[\mathrm{F} 2(\mathrm{MO})$ concur $\mathrm{E} 4(\mathrm{CH})>\mathrm{E} 3(\mathrm{YS})>\mathrm{E} 2$ $(\mathrm{NPSe})>\mathrm{E} 1(\mathrm{SA})]$ exceeded $[\mathrm{F} 3(\mathrm{NM})$ concur E4 $>$ E3 $>$ E2 $>$ E1] exceeded [F3 $(\mathrm{NM})$ concur F4 $>$ F3 $>$ F2 $>$ E1] exceeded [F2 (HA) concur E4 $>$ E3 $>$ E1] exceeded $[\mathrm{F} 1(\mathrm{NPK})$ concur E4 $>$ E3 $>$ F2 $>$ E1. These results were attributed to that significant promoting for growth traits that have been declared in Table 1. In despite, that there is no pest and microbial discusses incidence in the field experiment (except for NPKEo control) in both two seasons without using any agrochemical pesticide and miocrobiocide (except for npkwo control) which in consequence to biotic/abiotic elicitors under investigation-since, these elicitor trigger plants to induce systemic resistance (ISR)by regulating the expression of genes involved for production secondary metabolites (SMs) phytoalexins (PAs) which non-specific toxins against microbial deceases and pest infestation [42] [45] [57] that cause huge loss in yield production [44] under chemical fertilizer. Also abiotic/biotic elicitation evoked enhancing SMs production and quality [38]

Table 2. Mean seed yield traits values for 2 years (2017-2018) and (2018-2019) for $N$-sativa in response to 5 elicitors (F1-4) under 4 -fertilizers (E1-4).

\begin{tabular}{|c|c|c|c|c|c|}
\hline $\begin{array}{c}\text { Application } \\
\text { Treatments (FE) }\end{array}$ & $\begin{array}{l}\text { Dray seed yield } \\
\mathrm{g} / \mathrm{m}^{2}\left(\mathrm{DST}, \mathrm{g} / \mathrm{m}^{2}\right)\end{array}$ & $\begin{array}{l}\text { Fixed seed oil \% } \\
\text { (FO\%) }\end{array}$ & $\begin{array}{l}\text { Fixed seed oil, } \mathrm{g} / \mathrm{m}^{2} \\
\quad\left(\text { FOY }, / \mathrm{m}^{2}\right)\end{array}$ & $\begin{array}{l}\text { Essential seed oil } \\
\text { yield \% (EOY\%) }\end{array}$ & $\begin{array}{c}\text { Essential seed oil } \\
\text { yield, } \mathrm{g} / \mathrm{m}^{2} \\
\left(\text { EOY, } \mathrm{g} / \mathrm{m}^{2}\right)\end{array}$ \\
\hline \multicolumn{6}{|l|}{ NPK control } \\
\hline F1E0 & $168.500(+)$ & 31.25 & $52.653(+)$ & $1.212(+)$ & $2.040(+)$ \\
\hline F1E1 & $193.775(15.0)$ & 31.29 & $60.007(13.9)$ & $1.358(12)$ & $2.632(28.8)$ \\
\hline F1E2 & $198.830(18.0)$ & 31.31 & $61.637(17.1)$ & $1.406(16)$ & 2.789 (36.6) \\
\hline F1E3 & $202.200(20.0)$ & 31.25 & $62.682(19.0)$ & $1.515(25)$ & $3.063(50.0)$ \\
\hline F1E4 & $205.570(22.0)$ & 31.25 & $63.726(21.0)$ & $1.535(29)$ & $3.156(54.6)$ \\
\hline \multicolumn{6}{|l|}{ MOIE } \\
\hline F2E1 & $210.625(25.0)$ & 31.31 & $65.294(24.0)$ & $1.527(26)$ & $3.216(57.6)$ \\
\hline F2E2 & $212.310(26.0)$ & 31.27 & $65.816(25.0)$ & $1.600(32)$ & 3.397 (66.5) \\
\hline F2E3 & $217.365(29.0)$ & 31.25 & $67.383(28.0)$ & $1.648(36)$ & $3.582(75.0)$ \\
\hline F2E4 & $225.790(34.0)$ & 31.25 & $69.996(32.9)$ & $1.709(41)$ & 3.859 (89.2) \\
\hline \multicolumn{6}{|l|}{ NMLE } \\
\hline F3E1 & $208.098(23.5)$ & 31.27 & $64.510(22.5)$ & $1.467(21)$ & 3.053 (49.5) \\
\hline F3E2 & $208.940(24.0)$ & 31.29 & $64.771(23.0)$ & $1.503(24)$ & $3.140(53.8)$ \\
\hline F3E3 & $213.995(27.0)$ & 31.32 & $66.338(26.0)$ & $1.576(30)$ & $3.373(65.2)$ \\
\hline F3E4 & 220.735 (31.0) & 31.25 & $68.428(30.0)$ & $1.673(38)$ & $3.693(80.9)$ \\
\hline \multicolumn{6}{|l|}{ HA } \\
\hline F4E1 & $206.413(22.5)$ & 31.25 & $63.988(21.5)$ & $1.418(17)$ & 2.927 (43.3) \\
\hline F4E2 & $207.255(23.0)$ & 31.31 & $64.249(22.0)$ & $1.452(20)$ & 3.009 (47.4) \\
\hline F4E3 & $209.783(24.5)$ & 31.25 & $65.033(23.5)$ & $1.539(27)$ & $3.229(58.1)$ \\
\hline F4E4 & $215.680(28.0)$ & 31.25 & $66.861(27.0)$ & $1.612(33)$ & $3.477(70.3)$ \\
\hline LSD $1 \%$ & 0.541 & - & 0.225 & 0.007 & 0.004 \\
\hline
\end{tabular}

E0-4; control, salicylic acid (SA), nano-selenium oxide (SeN), yeast (Y), chitosan (CHT), E0 control respectively. F1-4; NPK, Moringa dry leaf extract (MOLE), Neem dry leaf extract (NMLE), humic acid (HA), respectively. Values between parenthesis (were percent of control). 


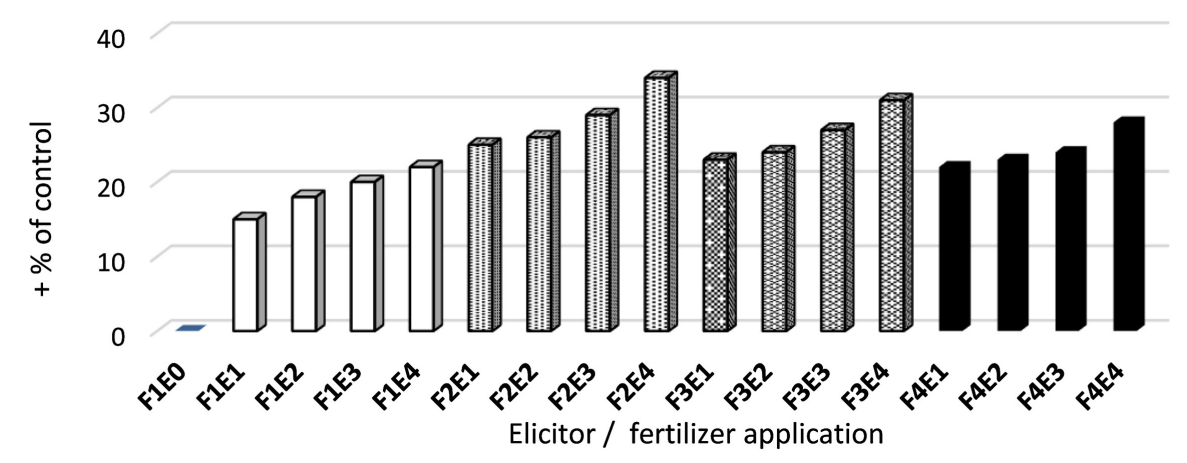

Figure 1. Dray seed yield, g/m² impacted with 4-elicitors (E1-4) under 4-fertilizers (F1-4).

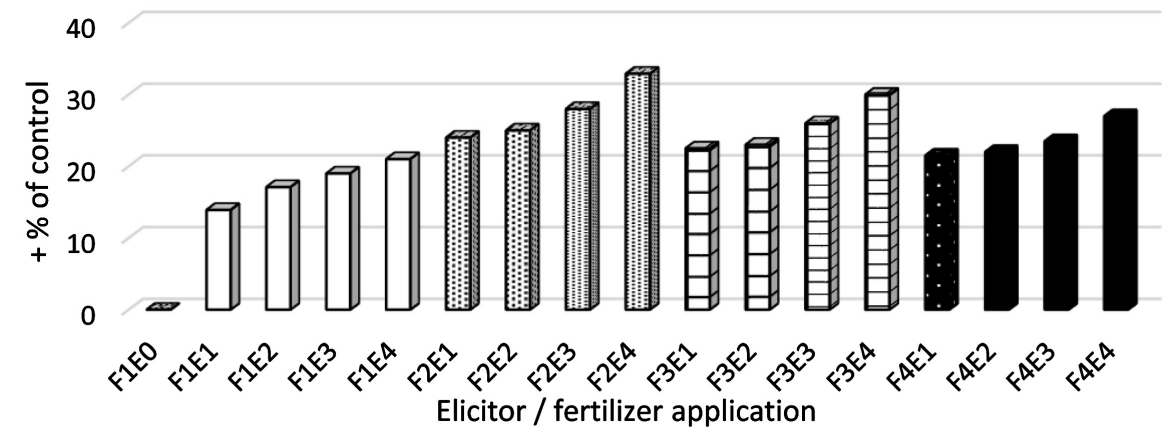

Figure 2. Fixed oil yield, g/m² impacted with 4-elicitors (E1-4) under 4-fertilizers (F1-4).

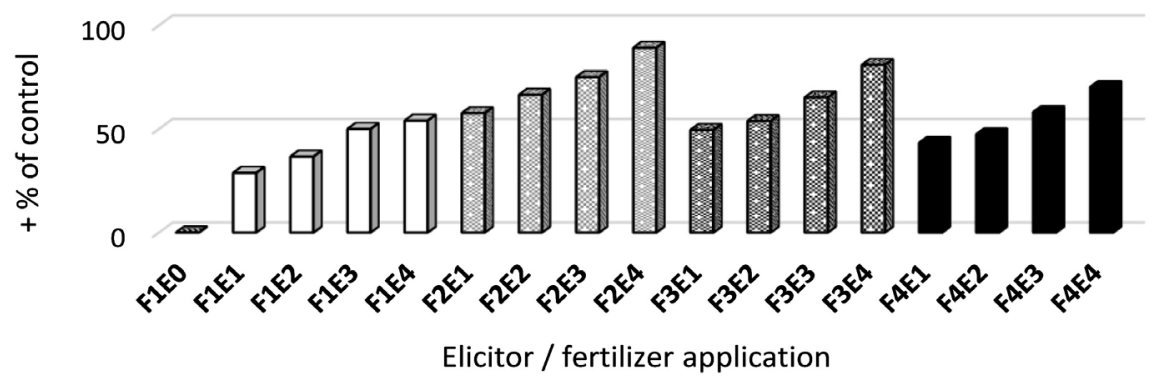

Figure 3. Essential oil yield, g/m² impacted with 5-elicitors (E1-4) under 4-fertilizers (F1-4).

[53] [58] [59] [60] [61] that overcoming biotic and abiotic stresses. Exceedingly chitosan (E4) proved bio-fungicide, bio-nemiotoside, bio-viroside [44] [62]. Also, SA elicitor enhanced growth and development and increased significantly [63] and improvement EO contents in organic agriculture system [64] MO effect on growth and yield of crops and thus can be promoting as a possible sublenent or substitute to inorganic fertilizers [59] these investigations confirmed our results shown in Table 1 and Table 2.

\subsection{Qualitative Yield Traits}

\subsubsection{Seed Fixed Oil (SFO)}

The major compounds SFO (Table 3) were comprised; lenoleinic (40.15\%), carvon (36.21\%), dihydroxylenolenic (21.12\%) and oleic (12.75\%) for NPK (F1E0) control. At such biofertilizer; MO (F2), NM (F3), HA (F4) concur with 
Table 3. Proportions of $N$. sativa fixed oil constituents impacted by four elicitors (E1-4) and three fertilizers (F1-4) (an average over of the two years experiment).

\begin{tabular}{|c|c|c|c|c|c|c|c|c|}
\hline \multirow{2}{*}{$\begin{array}{l}\text { Application treatment } \\
\text { (F/E) }\end{array}$} & \multicolumn{8}{|c|}{ Fatty acid 1 components, (\%) } \\
\hline & Linoleic & Oleic & Dihydrolelenic & Carvone & B.pinene & Nerole & Estragole & Carvacrole \\
\hline NPK control & $40.15(100)$ & $12.75(100)$ & $21.12(100)$ & $36.21(100)$ & 1.30 & 1.35 & 1.91 & 2.61 \\
\hline FIEO & $49.38(123)$ & $13.77(108)$ & $25.55(121)$ & $45.26(125)$ & 1.36 & 1.42 & 1.95 & 2.66 \\
\hline F1E1 & $54.20(135)$ & $14.28(112)$ & $26.40(125)$ & $46.34(128)$ & 1.43 & 1.48 & 1.97 & 2.68 \\
\hline F1E2 & $57.01(142)$ & $14.66(115)$ & $27.66(131)$ & $48.88(135)$ & 1.50 & 1.55 & 1.99 & 2.71 \\
\hline $\begin{array}{l}\text { F1E3 } \\
\text { F1E4 }\end{array}$ & $60.62(151)$ & $15.04(118)$ & $28(30(134)$ & $50.33(139)$ & 1.56 & 1.62 & 2.01 & 2.69 \\
\hline MOLE & $59.02(147)$ & $16.44(129)$ & $28.93(137)$ & $55.03(152)$ & 1.69 & 1.76 & 2.09 & 2.65 \\
\hline F2E1 & $63.43(158)$ & $17.46(137)$ & $30.62(145)$ & $56.84(157)$ & 176 & 1.82 & 2.13 & 2.66 \\
\hline F2E2 & $65.44(163)$ & $17.97(141)$ & $33.36(158)$ & $60.83(168)$ & 1.89 & 1.96 & 2.15 & 2.68 \\
\hline $\begin{array}{l}\text { F2E3 } \\
\text { F2E4 }\end{array}$ & 71.18 (179) & 18.99 (149) & 35.27 (167) & $61.91(171)$ & 1.95 & 1.63 & 2.06 & 2.65 \\
\hline NMLE & $57.81(144)$ & $15.42(121)$ & $28.51(135)$ & $53.59(143)$ & 1.63 & 1.69 & 2.04 & 2.63 \\
\hline F3E1 & $62.28(155)$ & $\begin{array}{l}15.42(121) \\
15.93(125)\end{array}$ & $\begin{array}{l}28.51(135) \\
29.56(140)\end{array}$ & $54.31(150)$ & $\begin{array}{l}1.63 \\
1.68\end{array}$ & $\begin{array}{l}1.69 \\
1.74\end{array}$ & $\begin{array}{l}2.04 \\
2.05\end{array}$ & $\begin{array}{l}2.63 \\
2.71\end{array}$ \\
\hline F3E2 & $64.64(161)$ & $16.57(130)$ & 31.04 (147) & 55.40 (153) & 1.76 & 1.82 & 2.06 & 2.67 \\
\hline $\begin{array}{l}\mathrm{F} 3 \mathrm{E} 3 \\
\mathrm{~F} 3 \mathrm{E} 4\end{array}$ & $67.45(168)$ & $17.21(135)$ & $31.89(151)$ & $57.21(158)$ & 1.82 & 1.89 & 1.96 & 2.65 \\
\hline HA & $55.40(138)$ & $15.04(118)$ & $26.40(125)$ & $47.07(130)$ & 1.57 & 1.63 & 1.67 & 2.61 \\
\hline F4E1 & $57.41(143)$ & $15.30(120)$ & $28.93(137$ & $48.52(134)$ & 1.65 & 1.72 & 1.68 & 2.66 \\
\hline F4E2 & $59.82(149)$ & $16.12(124)$ & $29.35(139)$ & $53.95(149)$ & 1.67 & 1.74 & 1.68 & 2.68 \\
\hline $\begin{array}{l}\text { F4E3 } \\
\text { F4E4 }\end{array}$ & $61.72(153)$ & $16.57(130)$ & 31.25 (148) & $54.67(151)$ & 1.69 & 1.76 & 1.79 & 2.64 \\
\hline LSD $1 \%$ & 0.25 & 0.08 & 0.15 & 0.21 & - & - & - & - \\
\hline
\end{tabular}

E0-4; control, salicylic acid (SA), nano-selenium oxide (SeN), yeast (Y), chitosan (CHT), EO control respectively. F1-4; NPK, Moringa dry leaf extract (MOLE), Ne. em dry leaf extract (NMLE), humic acid (HA), respectively. Values between parenthesis (were percent of control).

bioelicitors. $\mathrm{CH}$ (E4), YS (E3) and abiotic elicitors, NPSe (E2), SA (E1) performed significant increment in the four major components for SFO over that of traditional chemical fertilizer [NPK (F1)] coincide with non-elicitor control (E0). Aside, it follows that, [MO (F2) coincide CH (E4) > YS (E3) > NPSe (E2) > SA $(\mathrm{E} 1)>\mathrm{NPK}$ E0] excel $[\mathrm{NM}(\mathrm{F} 3)$ coincide E4 $>$ E3 $>$ E2 $>$ E1 $>$ NPK $(\mathrm{F} 1 \mathrm{E} 0)]$ excel [HA (F4) coincide E4 $>$ E3 $>$ E2 $>$ E1 $>$ NPK (F1) E0] excel [NPK F1 coincide E4 $>$ E3 $>$ E2 $>$ E1 $>$ NPK (F1) E0] Based on that biofertilizer concur bioelicitors excel significantly biofirtilizer coincide abiotic elicitor which were exceeded significantly over that traditional chemical fertilizer NPK (F1) under Zero-elicitor (E0) control, improving SFO quality consequently promoting health benefits.

\subsubsection{Seed Essential Oil (SE0)}

The major SEO contents (Table 4) were comprising; lamonine (39.45\%), Thymoquione, TQ (30.51\%), Penine, (10.55\%) for control, NPKE0 (Table 1). Biotic/abiotic elicitors inseparable biofertilizer and traditional chemical NPK fertilizer performed significant positive impacts for the four major components of 
Table 4. Proportions of $N$. sativa essential oil constituents impacted by five elicitors (E1-4) and four fertilizers (F1-4) (an average over of the two years experiment).

\begin{tabular}{|c|c|c|c|c|c|c|c|}
\hline \multirow{2}{*}{$\begin{array}{l}\text { Application treatment } \\
\qquad(\mathrm{F} / \mathrm{E})\end{array}$} & \multicolumn{7}{|c|}{ Essential oil components, as values per $100 \mathrm{~g}$. Seed (\%) } \\
\hline & pinene & Limonene & p. cymene & Carvacrol & $\begin{array}{c}\text { Thnymoquione } \\
\text { (TQ) }\end{array}$ & Sransaethole & Longifolene \\
\hline & $10.55(100)$ & 1.35 & $39.45(100)$ & 1.25 & $30.51(100)$ & 1.37 & 1.65 \\
\hline F1E0 & $11.71(111)$ & 1.37 & $41.42(105)$ & 1.26 & $35.69(117)$ & 1.38 & 1.62 \\
\hline FIEI & $12.02(114)$ & 1.38 & $42.60(108)$ & 1.27 & $36.61(120)$ & 1.39 & 1.66 \\
\hline $\begin{array}{ll}\text { FIE2 } \\
\text { F1F3 }\end{array}$ & $12.44(118)$ & 1.40 & $43.39(110)$ & 1.20 & $38.13(125)$ & 1.38 & 1.67 \\
\hline $\begin{array}{l}\text { F1E3 } \\
\text { F1E4 }\end{array}$ & $12.97(123)$ & 1.42 & $44.57(113)$ & 1.29 & $39.35(129)$ & 1.39 & 1.65 \\
\hline \multicolumn{8}{|l|}{ MOKE } \\
\hline $\begin{array}{ll}\mathrm{F} 2 \mathrm{E} 1 \\
\mathrm{~F} 2 \mathrm{~F} ?\end{array}$ & $14.87(141)$ & 1.46 & $46.55(118)$ & 1.30 & $47.29(155)$ & 1.41 & 1.65 \\
\hline F2E2 & $14.50(147)$ & 1.45 & $48.12(122)$ & 1.31 & $49.12(163)$ & 1.41 & 1.68 \\
\hline $\begin{array}{l}\text { F2E3 } \\
\text { F2F4 }\end{array}$ & $15.93(151)$ & 1.47 & $49.31(125)$ & 1.32 & $50.34(165)$ & 1.39 & 1.67 \\
\hline \multicolumn{8}{|l|}{ NMLE } \\
\hline F3E1 & $13.82(131)$ & 1.39 & $46.15(117)$ & 1.28 & $44.23(145)$ & 1.40 & 1.62 \\
\hline $\mathrm{F} 3 \mathrm{E} 2$ & 14.55 & 1.40 & $46.94(119)$ & 1.29 & $45.15(148)$ & 1.39 & 1.61 \\
\hline $\begin{array}{l}\text { F3E3 } \\
\text { F3E4 }\end{array}$ & $14.98(142)$ & 1.41 & $47.73(121)$ & 1.26 & $46.37(152)$ & 1.39 & 1.66 \\
\hline \multicolumn{8}{|l|}{$\mathrm{HA}$} \\
\hline F4E1 & $13.60(129)$ & 1.40 & $44.97(114)$ & 1.30 & $41.18(135)$ & 1.40 & 1.67 \\
\hline F4E2 & $13.71(130)$ & 1.44 & 45.76 (116) & 1.29 & $42.10(138)$ & 1.37 & 1.68 \\
\hline $\begin{array}{l}\text { F4E3 } \\
\text { F4E4 }\end{array}$ & $14.24(135)$ & 1.40 & 46.15 (117) & 1.27 & $44.23(145)$ & 1.38 & 1.66 \\
\hline LSD $1 \%$ & 0.06 & - & 0.21 & - & 0.17 & - & - \\
\hline
\end{tabular}

E0-4; control, salicylic acid (SA), nano-selenium oxide (SeN), yeast (Y), chitosan (CHT), E0 control respectively. F1-4; NPK, Moring a dry leaf extract (MOLE), Neem dry leaf extract (NMLE), humic acid (HA), respectively. Values between parenthesis (were percent of control).

SEO (Table 1). Aside, [MO (F2) coincur; $\mathrm{CH}(\mathrm{E} 4)>\mathrm{YS}(\mathrm{E} 3)>\mathrm{NPSe}(\mathrm{E} 2)>\mathrm{SA}$ (E1) $>\mathrm{NM}(\mathrm{F} 3)$ concur; $\mathrm{CH}(\mathrm{E} 4)>\mathrm{YS}(\mathrm{E} 3)>\mathrm{NPSe}(\mathrm{E} 2)>\mathrm{SA}(\mathrm{E} 1)]>\mathrm{HA}(\mathrm{F} 1)$ concur, $\mathrm{CH}(\mathrm{E} 4)>\mathrm{YS}(\mathrm{E} 3)>\mathrm{NPse}(\mathrm{E} 2>\mathrm{SA}(\mathrm{E} 1)]>\mathrm{NPK}(\mathrm{F} 1)$ concur $\mathrm{CH}(\mathrm{E} 4)>$ YS (E3 ) > NPSe (F2) > SA (E1)] therefore biotic elicitors; CH (E4), YS (F3) exceed significantly abiotic elicitors; NPSe (E2), SA(E1), concur biofertilizer; MO (F2), NM (F3), HA (F2), which excel significantly NPK(F1)E0 to improve SEO quality and ameliorated health promoting benefits Extensing study for elicitation revealed upraise EOs production and quality undertrditional chemical fertilizers [43] [59]. Also solitary biofertilizer application have been shown the positive impacts of EOs quality and quantity [65] [66] the different EOs compositions and different mutal ratio of compounds contained in EOs may excert significant on their biological efficacy and their components demonstrated beneficial impacts on human health, antimicrobial, antifungal, antiviral and food preservation [67] [68]. Also, the resultant increment in the main major component of SFO and SEO was more or less in line with what has been reported recently [4] [69]. 


\section{Conclusion}

Based on overall obtained, it could be considered multi-repeating biotic/abiotic elicitation correlative biofertilizers as a reliable technological strategy to boast up significantly $N$. sativa, dray seed, seed fixed oil and seed essential oil yield production. Alongside, ameliorating significantly bioactive compound contents for both seed fixed, essential oils. Consequently, highlight to achieve sustainable development for $N$. sativa under organic and traditional inorganic system.

\section{Conflicts of Interest}

The authors declare no conflicts of interest regarding the publication of this paper.

\section{References}

[1] Gilani, A., Jabeen, Q. and Ullah khan, M. (2004) A Review of Medicinal Uses and Pharmacological Activities of Nigella sativa. Pakistan Journal of Biological Sciences, 7, 441-451. https://doi.org/10.3923/pjbs.2004.441.451

[2] Subhash, P., Sanjeev, B., Aami, A., Ramzi, M. and Fazlul, H.S. (2008) From Here to Eternity the Secret of Pharaohs: Therapeutic Potential of Black Cumin Seeds and Beyond. Cancer Therapy, 6, 495-510.

[3] Maskani, S., Tafazli, M., Rakshandeh, H., Esmaily, H. and Dadgar, S. (2019) The Effect of Nigella sativa Seeds Capsules on the Severity of Physical Symptoms People with Premenstrual Syndrome. Journal of Medicinal Plant, 18, 164-174, 196.

[4] Butt, A.S., Nisar, N., Ghani, N., Altaf, I. and Aziz Mughal, T. (2019) Isolation of Thymoquinone from Nigella sativa L. and Thymus vulgaris L., and Its Anti-Proliferative Effect on HeLa Cancer Cell Lines. Tropical Journal of Pharmaceutical Research, 18, 37-42. https://doi.org/10.4314/tjpr.v18i1.6

[5] Nergiz, C. and Otles, S. (1993) Chemical Composition of Ngella sativa L. Seeds. Food Chemistry, 48, 259-261. https://doi.org/10.1016/0308-8146(93)90137-5

[6] Ghosheh, O.A., Houdi, A.A. and Crooks, P.A. (1999) High Performance Liquid Chromatographic Analysis of the Pharmacologically Active Quinones and Related Compounds in the Oil of the Black Seed (Nigella sativa L.). Journal of Pharmaceutical and Biomedical Analysis, 19, 757-762. https://doi.org/10.1016/S0731-7085(98)00300-8

[7] Tavakkoli, A., Ahmadi, A., Razavi, B.M. and Hosseinzadeh, H. (2017) Black Seed (Nigella sativa L.) and Its Constituent Thymoquinone as an Antidote or a Protective Agent against Natural or Chemical Toxicities. Iranian Journal of Pharmaceutical Research, 16, 2-23.

[8] Hosseinzadeh, H. and Montahaei, R. (2007) Protective Effect of Nigella sativa L. Extracts and Thymoquinone, Its Active Constituent, on Renal Ischemia Reperfusion Induced Oxidative Damage in Rats. Pharmacology Online, 1, 176-189.

[9] Hajhashemi, V., Ghannadi, A. and Jafarabadi, H. (2004) Black Cumin Seed Essential Oil, as a Potent Analgesic and Antiinflammatory Drug. Phytotherapy Research, 18, 195-199. https://doi.org/10.1002/ptr.1390

[10] Amin, B., Taheri, M.M.H. and Hosseinzadeh, H. (2014) Effects of Intraperitoneal Thymoquinone on Chronic Neuropathic Pain in Rats. Planta Medica, 80, 1269-1277. https://doi.org/10.1055/s-0034-1383062 
[11] Hosseinzadeh, H., Eskandari, M. and Ziaee, T. (2008) Antitussive Effect of Thymoquinone, a Constituent of Nigella sativa Seeds, in Guinea Pigs. Pharmacology Online, 2, 480-484.

[12] Leong, X.F., Rais Mustafa, M. and Jaarin, K. (2013) Nigella sativa and Its Protective Role in Oxidative Stress and Hypertension. Evidence-Based Complementary and Alternative Medicine, 2013, Article ID: 120732.

https://doi.org/10.1155/2013/120732

[13] Kanter, M., Meral, I. and Yener, Z. (2003) Partial Regeneration/Proliferation of the Beta-Cells in the Islets of Langerhans by Nigella sativa L. in Streptozotocin-Induced Diabetic Rats. The Tohoku Journal of Experimental Medicine, 201, 213-219.

https://doi.org/10.1620/tjem.201.213

[14] Rakhshandeh, H., Vahdati-Mashhadian, N. and Khajekaramadini, M. (2011) In-Vitro and In-Vivo Study of the Antibacterial Effects of Nigella sativa Methanol Extract in Dairy Cow Mastitis. Avicenna Journal of Phytomedicine, 1, 29-35.

[15] Koka, P., Mondal, D., Schultz, M., Abdel-Mageed, A. and Agrawal, K. (2010) Studies on Molecular Mechanisms of Growth Inhibitory Effects of Thymoquinone against Prostate Cancer Cells: Role of Reactive Oxygen Species. Experimental Biology and Medicine, 235, 751-760. https://doi.org/10.1258/ebm.2010.009369

[16] Butt, A.S., Nisar, N., Ghani, N., Aitaf, I. and Mughal, T.A. (2019) Isolation of Thymoquinone from Nigella sativa L. and Thymus vulgaris L., and Its Anti-Proliferative Effect on Hela a Cancer Cell Lines. Tropical Journal of Pharmaceutical Research, 18, 37-42. https://doi.org/10.4314/tjpr.v18i1.6

[17] Hosseinzadeh, H., Parvardeh, S., Nassiri-Asl, M. and Mansouri, M.-T. (2005) Intracerebroventricular Administration of Thymoquinone, the Major Constituent of $\mathrm{Ni}$ gella sativa Seeds, Suppresses Epileptic Seizures in Rats. Medical Science Monitor, 11, BR106. https://doi.org/10.1078/0944-7113-00376

[18] Parvardeh, S., Nassiri-Asl, M., Mansouri, M.T. and Hosseinzadeh, H. (2005) Study on the Anticonvulsant Activity of Thymoquinone, the Major Constituent of Nigella sativa L. Seeds, through Intracerebroventricular Injection. Journal of Medicinal Plants, 4, 45-52.

[19] Mehri, S., Shahi, M., Razavi, B.M., Hassani, F.V. and Hosseinzadeh, H. (2014) Neuroprotective Effect of Thymoquinone in Acrylamide Induced Neurotoxicity in Wistar Rats. Iranian Journal of Basic Medical Sciences, 17, 1007-1011.

[20] Nagi, M. and Mansouru, M. (2000) Protective Effect of Thymoquinone against Doxorubicin Induced Cardiotoxicity in Rats: A Possible Mechanism of Protection. Pharmaceutical Research, 41, 283-289. https://doi.org/10.1006/phrs.1999.0585

[21] Nagi, M.N., Alam, K., Badary, O.A., Al-Shabanah, O.A., Al-Sawaf, H.A. and Al-Bekairi, A.M. (1999) Thymoquinone Protects against Carbon Tetrachloride Hepatotoxicity in Mice via an Antioxidant Mechanism. Biochemistry and Molecular Biology International, 47, 153-159. https://doi.org/10.1080/15216549900201153

[22] Boskabady, M. and Farhadi, F. (2008) The Possible Prophylactic Effect of Nigella sativa Seed Extract on Respiratory Symptoms, Pulmonary Function Tests on Chemical War Victims. Journal of Alternative and Complementary Medicine, 14, 1137-1144. https://doi.org/10.1089/acm.2008.0049

[23] Hosseinzadeh, H., Taiari, S. and Nassiri-Asl, M. (2012) Effect of Thymoquinone, a Constituent of Nigella sativa L., on Ischemia-Reperfusion in Rat Skeletal Muscle. Archives of Pharmacology, 385, 503-508.

https://doi.org/10.1007/s00210-012-0726-2 
[24] Ahmad, A., Husain, A., Mujeeb, M., Khan, S.A., Najmi, A.K., Siddique, N.A., et al. (2013) A Review on Therapeutic Potential of Nigell sativa: A Miracle Herb. Asian Pacific Journal of Tropical Biomedicine, 5, 337-352. https://doi.org/10.1016/S2221-1691(13)60075-1

[25] Woo, C.C., Loo, S.Y., Gee, V., Yap, C.W., Sethi, G. and Kumar, A.P. (2011) Anticancer Activity of Thymoquinone in Breast Cancer Cells: Possible Involvement of PPAR- $\gamma$ Pathway. Biochemical Pharmacology, 82, 464-475.

https://doi.org/10.1016/j.bcp.2011.05.030

[26] Mukhtar, H., Qureshi, A.S., et al. (2019) Nigella sativa.

[27] Gomaez-candela, C., Bermejo Lopez, L.M. and Loria-Kohen, V. (2011) Importance of a Balwnced Omega 6/Omega 3 Ratio for the Maintenance of Health Nutritional Recommendations. Nutricion Hospitalaria, 26, 329.

[28] Tvrzicka, E., Kremmyda, L.-S., Stankova, B., Zak, A. and Republic, C. (2011) Fatty Acids as Biocompounds: Their Role in Human Metabolism, Health and Disease: A Review. Part 1: Classification, Dietary Sources and Biological Functions. Biomedical Papers of the Medical Faculty of the University Palacky, Olomouc, Czechoslovakia, 155, 117-130. https://doi.org/10.5507/bp.2011.038

[29] Lin, T.Y. (2006) Conjugated Linoleic Acid Production by Cells and Enzyme Extract of Lactobacillus delbrueckii ssp. Bulgaricus with Additions of Different Fatty Acids. Food Chemistry, 94, 437-441. https://doi.org/10.1016/j.foodchem.2004.11.032

[30] Hennessy, A.A. (2009) BIOCLA: Biotechnological Approaches for the Development of Functional Foods Enriched in Bioactive Lipids. 1-19.

[31] Calder, P.C. and Yaqoob, P. (2009) Omega-3 Polyunsaturated Fatty Acids and Human Health Outcomes. Biofactors, 35, 266-272. https://doi.org/10.1002/biof.42

[32] Ruxton, C.H.S., Calder, P.C., Reed, S.C. and Simpson, M.J.A. (2005) The Impact of Long-Chain n-3 Polyunsaturated Fatty Acids on Human Health. Nutrition Research Reviews, 18, 113-129. https://doi.org/10.1079/NRR200497

[33] Simopoulos, A.P. (2008) The Importance of the Omega-6/Omega-3 Fatty Acid Ratio in Cardiovascular Disease and Other Chronic Diseases. Experimental Biology and Medicine, 233, 674-688. https://doi.org/10.3181/0711-MR-311

[34] Angelova, Z., Georgiev, S. and Roos, W. (2006) Elicitation of Plants. Biotechnology \& Biotechnological Equipment, 20, 72-83. https://doi.org/10.1080/13102818.2006.10817345

[35] Świeca, M. (2016) Elicitation and Treatment with Precursors of Phenolics Synthesis Improve Low-Molecular Antioxidants and Antioxidant Capacity of Buckwheat Sprouts. Acta Scientiarum Polonorum, Technologia Alimentaria, 15, 17-28. https://doi.org/10.17306/J.AFS.2016.1.2

[36] Sharma, R.Z. (2016) Optimization of Methyl Jasmonate and -cyclodextrinrenhanced Taraxerol and Taraxasterol in (Taraxacumofficinale Weber) Cultures. Plant Physiology and Biochemistry, 103, 24-30. https://doi.org/10.1016/j.plaphy.2016.02.029

[37] Mohammad parast, B., Rasoul, M., Rustaiee, A.R., Zardari, S. and Agrawel, V. (2014) Quantification of Asiatic Acid from Plant Parts of Centella asiatic L. and Enhancement of Its Synthesis through Organic Elicitors in In-Vitro. Horticulture, Environment and Biotechnology, 55, 578-582. https://doi.org/10.1007/s13580-014-0168-5

[38] Mejdoub-Trabelsi, B., Touihri, S., Ammar, N., Riahi, A. and Daami-Remadi, M. (2020) Effect of Chitosan for the Control of Potato Diseases Caused by Fusarium Species. Journal of Phytopathology, 168, 18-27. https://doi.org/10.1111/jph.12847 
[39] Ahamed, T.E.S. (2019) Bioprospecting Elicitation with Gamma Irradiation Combine with Chitosan to Enhance, Yield Production, Bioactive Secondary Metabolites and Antioxidant Activity for Saffron. Journal of Plant Sciences, 7, 137-143.

[40] Gao, M.B., Zhang, W., et al. (2011) Significantly Improved Taxuyunnanine C Production in Cell Suspension Culture of Taxus chinensis by Process Intensification of Repeated Elicitation, Sucrose Feeding, and in Situ Adsorption. World Journal of Microbiology and Biotechnology, 27, 2271-2279.

https://doi.org/10.1007/s11274-011-0690-x

[41] Sharifi-Rad, M., Sharifi-Rad, J.A. and da Silva, T. (2016) Morphological, Physiological and Biochmical Rerponses of Crops (Zea mays L., Phaseolus vulgaris L.), Medicinal Plants (Hyssopus officinalis L., Nigella sativa L.) and Weeds (Amaranthus retroflexus L., Taraxacum officinale F.H. Wigg) Exposed to $\mathrm{SIO}_{2}$ Nanoparticles. Journal of Agriculture, Science and Technology, 18, 1027-1040.

[42] Jalali, S.M., Movahhedi, D.M., Salehi, A. and Bahreininejad, B. (2019) Effect of Irrigation Regimes and Nitrogen Sources on Biomass Production, Water and Nitrogen Use Efficiency and Nutrients Uptake in Coneflower (Echinacea purpurea L.). Agricultural Water Management, 213, 358-367. https://doi.org/10.1016/j.agwat.2018.10.011

[43] Ahamed, T.E.S. and El-Sayed, S.A. (2018) Verification and Validation of Dandelion (Taraxacum officinal) Seeds-Gamma Irradiated under Elicitation with Nano- and Micro-Zinc for Potential Optimization Biomass and Ennghanci Phenolics, Flavonoids and Antioxidant Activity. International Journal of Innovative Science and Research Technology, 3, 398-403.

[44] Zheng, L., Hong, F.S., Lu, S.P. and Liu, C. (2005) Effect of Nano-Tio ${ }_{2}$ on Strength of Naturally and Growth Aged Seeds of Spinach. Biological Trace Element Research, 104, 83-91. https://doi.org/10.1385/BTER:104:1:083

[45] Adam, A., Jourdan, E., Pgena, M., Duby, F., Domes, J. and Thonart, P. (2005) Resistance Induced in Cucumber and Tomato by a Non-Pathogenic Pseudomonas putida Strain. Parasitica, 61, 13-22.

[46] Gabaston, J., EL-Khawand, T., Waffo-Teguo, P., Decendit, A., Richard, T., Merillon, J.M. and Pavela, R. (2018) Stilbenes from Grapevine Root: A Promising Natural Insecticide against Leptinotarsa decemlineata. Journal of Pest Science, 91, 897-906. https://doi.org/10.1007/s10340-018-0956-2

[47] Hassan, M. and Bae, H.H. (2017) An Overview of Stress-Induced Resveratrol Synthesis in Grapes: Perspectives for Resveratrol-Enriched Grape Products. Molecules, 22, 294. https://doi.org/10.3390/molecules22020294

[48] Jansen, M.A.K., Hectors, K., O’Brien, N.M., Guisez, Y. and Potters, G. (2008) Plant Stress and Human Health: Do Human Consumers Benefit from UV-B Acclimated Crops. Plant Science, 175, 449-458. https://doi.org/10.1016/j.plantsci.2008.04.010

[49] Edreva, A., Velikova, V., Tsonev, T., Dagnon, S., Gurel, A., Aktas, L. and Gesheva, E. (2008) Stress Protective Role of Secondary Metabolites: Diversity of Functions and Mechanisms. General and Applied Plant Physiology, 341, 67-78.

[50] Treutter, D. (2005) Significance of Flavonoids in Plant Resistance and Enhancement of Their Biosynthesis. Plant Biology, 7, 581-591. https://doi.org/10.1055/s-2005-873009

[51] Khoddami, A., Ghazali, H.M., Yassoralipour, A., Ramakrishnan, Y. and Ganjloo, A. (2011) Physicochemical Characteristics of Nigella Seed (Igella sativa L.) Oil as Affected by Different Extraction Methods. Journal of the American Oil Chemists' Society, 88, 533-540. https://doi.org/10.1007/s11746-010-1687-6 
[52] Hadad, G.M., Salam, R.A., Soliman, R.M. and Mesbah, M.K. (2012) High-Performance Liquid Chromatography Quantification of Principal Ant, Oxidants in Black Seed (Nigella sativa L.) Phytopharmaceuticals. Journal of AOAC International, 95, 1043-1047. https://doi.org/10.5740/jaoacint.11-207

[53] Ahamed, T.E.S. and El-Sayed, S.A. (2018) Practical Field Application for Elicitation Efficacy with Gamma Irradiation, Nano-Selenium-Oxide and/or Saccharomyces cerevisiae as Elicitors Mediated Amelioration Biomass, Flavonoids, Rutin and Antioxidant Capacity of Tartary Buckwheat. International Journal of Innovative Science and Research Technology, 3, 268.

[54] Costa, V.C.O. (2008) Chemical Composition and Modulation of Bacterium 1 Drug Resistance of the Essential Oil from the Leaves of Rollinia leptopetala R.E. Fries. Revista Brasileira de Farmacognosia, 18, 245-248.

https://doi.org/10.1590/S0102-695X2008000200019

[55] Sen, A., Khade, S.D., Jana, J.C. and Choudhury, P. (2019) Effect of Integrated Nutrient Management on Growth Yield and Quality Attributes of Black Cumin ( $\mathrm{Ni}$ gella sativa L.) var Rajendra Shyama Grown under Terai Region of West Bengal. Journal of Spices and Aromatic Crops, 28, 61-65.

[56] Smitha, G.R., Basak, B.B., Thondaiman, V. and Saha, A. (2019) Nutrient Management through Organic, Bio Fertilizers and Crop Residues Improves Growth Yield and Quality of Sacred Basil (Ocimum sanctum Linn). Industrial Crops and Products, 128, 599-606. https://doi.org/10.1016/j.indcrop.2018.11.058

[57] Yedidia, I., Shoresh, M., Kerem, Z., Benhamou, N., Kapulnik, Y. and Chet, I. (2003) Concomitant Induction of Systemic Resistance to Pseudomonas syringae pv. Lachrymans in Cucumber by Trichoderma asperellum (T-203) and Accumulation of Phytoalexins. Applied and Environmental Microbiology, 69, 7343-7353. https://doi.org/10.1128/AEM.69.12.7343-7353.2003

[58] Tavallali, V., Rowshan, V., Gholami, H. and Hojati, S. (2020) Iron-Urea Nano-Complex Improves Bioactive Compounds in Essential Oils of Ocimum basilicum L. Scientia Horticulturae, 265, Article ID: 109222. https://doi.org/10.1016/j.scienta.2020.109222

[59] Ahamed, T.E.S. and El-Sayed, S.A. (2020) Verifying Potential of Moringa oleifera Extract Application as Bio-Fertilizer for Basil Plants (Ocimum basilicum L.) Elicited with Gamma Irradiation and/or Nano-Zinc Oxide to Ameliorate Biomass Quantity and Quality. Asian Journal of Science and Technology, 11, 10888-10897.

[60] Kruszka, D., Sawikowska, A., Selvakesavan, R.K. and Krajewski, P. (2020) Silver Nanoparticles Affect Phenolic and Phytoalexin Composition of Arabidopsis thaliana. Science of the Total Environment, 716, Article ID: 135361. https://doi.org/10.1016/j.scitotenv.2019.135361

[61] Ahmad, Z., Khan, Q.U., Qadoos, A. and Khan, M.J. (2020) Humic Acid, an Effective Amendment Used for Amelioration of Phosphatic Fertilizer and Enhancing Maize Yield. Pure and Applied Biology, 9, 750-759.

https://doi.org/10.19045/bspab.2020.90081

[62] Vilela, J., Martins, D., Monteiro-Silva, F., Aguilar, G., Almeida, J. and Saraiva, C. (2016) Antimicrobial Effect of Essential Oils of Laurus nobilis L. and Rosmarinus officinallis L. on Shelf-Life of Minced Mayonesa Beef Stored under Different Packaging Conditions. Food Packaging and Shelf Life, 8, 71-80. https://doi.org/10.1016/j.fpsl.2016.04.002

[63] Abdi, G. and Karami, L. (2020) Salicylic Acid Effects on Some Physiochemical Properties and Secondary Metabolite Accumulation in Mentha piperita L. under 
Water Deficit Stress. Advances in Horticultural Science, 34, 81-91.

[64] Nasiri, Y., Zandi, H. and Morshedloo, M.R. (2018) Effect of Salicylic Acid and Ascorbic Acid on Essential Oil Content and Composition of Dragonhead (Dracocephalum moldavica L.) under Organic Farming. Journal of Essential Oil-Bearing Plants, 21, 362-373. https://doi.org/10.1080/0972060X.2018.1453383

[65] Azzaz, N.A., Hassan, E.A. and Hamad, E.H. (2009) The Chemical Constituent and Vegetative Yielding Characteristics of Fennel Plants Treated with Organic and Bio-Fertilizer Instead of Mineral Fertilizer. Australian Journal of Basic and Applied Sciences, 3, 579-587.

[66] El-Naggar, A.H.M., Hassan, M.R.A., Shaban, E.H. and Mohamed, M.E.A. (2015) Effect of Organic and Biofertilizers on Growth, Oil Yield and Chemical Composition of the Essential Oil of Ocimum basillicum L. Plants. Alexandria Journal of Agricultural Research, 60, 1-16.

[67] Roohinejad, S., Koubaa, M., Barba, F.J., Leong, S.Y., Khelfa, A., Greiner, R. and Chemat, F. (2017) Extraction Methods of Essential Oils from Herbs and Spices. In: Essential Oils in Food Processing, John Wiley \& Sons Ltd., Hoboken, 21-55. https://doi.org/10.1002/9781119149392.ch2

[68] Giacometti, J., Ević, D.B.K., Putnik, P., Gabrić, D., et al. (2018) Extraction of Bioactive Compounds and Essential Oils from Mediterranean Herbs by Conventional and Green Innovative Techniques: A Review. Food Research International, 113, 245-262. https://doi.org/10.1016/j.foodres.2018.06.036

[69] Kamalizadeh, M., et al. (2019) Drought Stress and $\mathrm{TiO}_{2}$ Nanoparticles Affect Composition of Different Active Compounds in Moldavian Dragonhead Plant. Acta Physiologiae Plantarum, 41, 2. https://doi.org/10.1007/s11738-019-2814-0 